\title{
VISUAL PROCESSING OF FOUR KINDS OF RELATIVE MOTION
}

\author{
D. REGAN \\ Daihousie University, Halifax, Nova Scotia, Canada B3J 1 B6
}

\begin{abstract}
Evidence is presented supporting the idea that the human visual system has several specific sensitivities to different kinds of relative motion. These specific sensitivities include: (1) sensitivity to a velocity difference between two different points $A$ and $B$ on one eye's retinal inage, the two velocities being directed along the line $\mathrm{AB}$; (2) sensitivity to the velocity difference at $\mathrm{A}$ and $\mathrm{B}$ between velocity components perpendicular to the line $A B$ (i.e. shearing motion); (3) sensitivity to rotary motion; (4) sensitivity to the ratio between the velocities of the left and right retinal images of an object that is moving in depth. These specific sensitivities can be attributed to relatively hardwired neural filters that are "tuned" to differenent retinal image correlates of the three-dimensional structure and motion of solid objects in the environment. Such filters may be of use in distinguishing rigid nonrotating objects from nonrigid or rotating objects. They may also be of use in recovering information from the two-dimensional retinal image, including information about object boundaries, the three-dimensional structure of the environment, self-motion and object motion in depth. An alternative way of regarding certain of these specific sensitivities is that they might provide rough physiological equivalents of the values of div $\mathbf{V}$ and curl $\mathbf{V}$ at every point in the instantaneous velocity field of the retinal image and thus crudely analyze the retinal image flow pattern in terms of mathematical quantities that have the useful property of being relatively invariant against bodily translations of the whole retinal image caused by eye rotation.
\end{abstract}

Vision Motion Stereopsis Figure-ground Retinal flow Image understanding

\section{INTRODUCTION}

A surface moving parallel to the frontal plane produces a retinal image which has the same velocity at every point on the image. Also, the retinal images in the left and right eyes move identically. There is an extensive literature on this kind of motion (Sekuler et al., 1978). Other kinds of motion, for example motion in depth, produce a retinal image which has different retinal image velocities at different points on the image, and in addition the image velocities in the left and right eyes may be different. It has been proposed that the visual system has several specific sensitivities to different kinds of relative motion and that these specific sensitivities may be of use in using motion to recover from the visual flow pattern information about the observer's self-motion and information about the three-dimensional structure of the environment.

This paper discusses visual processing of four kinds of relative motion. The first involves specific sensitivity to a difference in velocity at two different points $A$ and $B$ on the retina of one eye, the two velocities being directed along a straight line $A B$ [Fig. $1(A)$ ]. In the onedimensional case, this specific sensitivity may be involved in extracting figure from ground and in differentiating between rigid, nonrotating objects and nonrigid or rotating objects. In the two-dimensional case (i.e. looming), this specific sensitivity may be involved in recovering an object's motion in depth from its retinal image. The second sensitivity is to shearing motion, i.e. to differences in the velocity components at $A$ and $B$ along a line perpendicular to $A B$ [Fig. 1(B)]. This sensitivity may be involved in utilizing motion parallax to extract figure from ground. The third sensitivity is to rotary motion [Fig. 1(C)]. The fourth sensitivity is to the ratio between velocities in of an object's right and left retinal images. This sensitivity may be involved in accurately discriminating the direction of motion in depth, for example in judging whether a moving object will hit one's head and also in judging the speed of motion in depth.

It is suggested that visual sensitivities to looming and to rotary motion can be regarded 


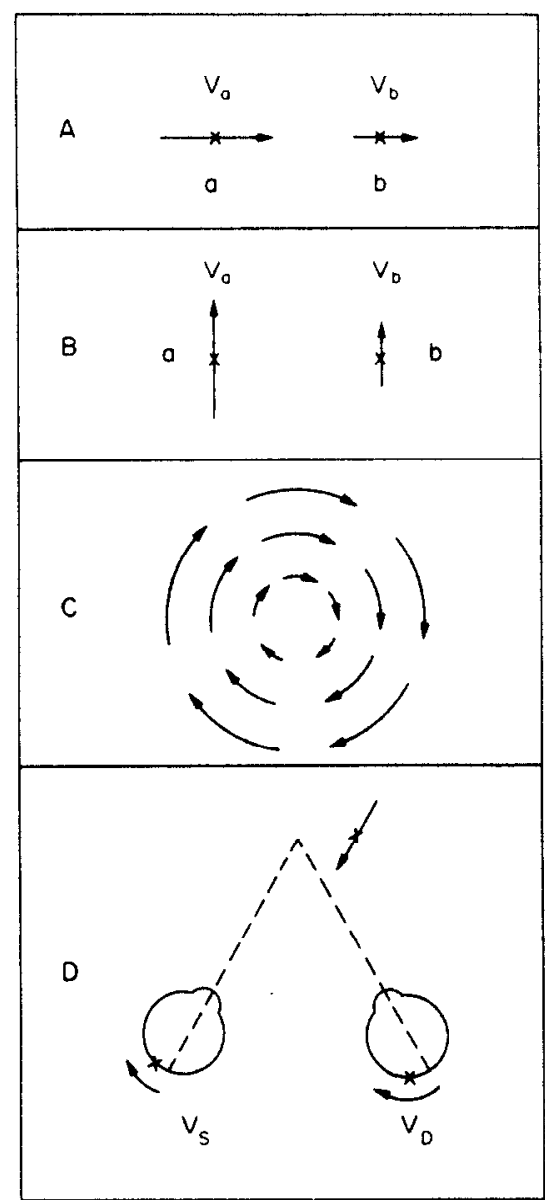

Fig. 1. $V_{a}$ and $V_{b}$ are the velocities at two points $a$ and $b$ on an object's retinal image. (A) The one-dimensional case that the velocity components along the line $a b$ are different at $a$ and $b$. The two-dimensional case (not illustrated) is size change or looming. (B) The velocity components perpendicular to the line $a b$ are different at $a$ and $b$ (i.e. shearing motion). (C) Rotary motion. (D) For an object moving in depth the left and right eye's retinal image velocities $V_{\mathrm{s}}$ and $V_{D}$ are different.

as being mediated by rough physiological equivalents to detectors of local div $\mathrm{V}$ and curl $\mathrm{V}$ respectively.

\section{SECTION}

Opponent-motion and relative-motion elements

In this section we discuss the kind of onedimensional relative motion illustrated in Fig.

\footnotetext{
"A hypothetical "neural element" is used here in the sense of the smallest neural organisation that can perform an information-filtering function. In principle, a higher element may be built up from simpler elements. For example, a two-dimensional looming element might be built up from one-dimensional opponent-motion elements (see below)
}

1(A) where the components of velocity along a straight line $a b$ are different at locations a and b. It has been suggested that the human visual system contains neural elements* sensitive to this kind of relative motion. Supporting evidence includes the finding that motion threshold is considerably higher for a target moving across a uniform homogenous background than for a target moving with respect to stationary reference marks (Kaufman, 1974). A second line of evidence derives from experiments in which the width (but not the height) of a bar is oscillated. Adapting to this stimulus reduces visual sensitivity to oscillations of bar width, but has little effect on the ability to detect translational movements of the bar, suggesting that the site of adaptation is where the signals from the moving edges interact (Beverley and Regan, 1980). An additional relevant finding is that, after adapting to a bar of constant height whose width repetitively increases with a ramping waveform, a subsequently-viewed bar of constant width appears to be shrinking in width. The crucial point here is that this negative aftereffect is much stronger than the classical motion aftereffect produced by the bar (Regan and Beverley, 1978b).

Relative motion elements whose function is equivalent to Fig. 2 have been proposed in order to account for the above findings. We supposed that the visual pathway contains opponentmotion elements sensitive to absolute retinal velocity that give a positive output for one direction of motion and a negative output for the opposite direction of motion, and that a one-dimensional relative motion element is built up from two opponent-motion elements (Regan and Beverley, 1978a, 1980). Opponent-motion elements that are excited by one direction of motion and inhibited by the opposite direction of motion have been proposed on several psychophysical grounds (Levinson and Sekuler, 1975; Nakayama and Tyler, 1981; Stronmeyer et al., 1984), and direction-selective neurons that are inhibited by the nonpreferred direction of motion are well known (Maturana and Franck, 1963; Emerson and Gerstein, 1977; Movshon et al., 1978; Goodwin et al., 1975; Goodwin and Henry, 1975; Michael, 1966; Barlow and Levick, 1965). We further supposed that the output of an opponent-motion element is proportional to speed [Fig. 2(A)]. Consider two of these opponent-motion elements (M1 and M2) with the same preferred direction of motion, and driven from adjacent retinal locations so that 
A

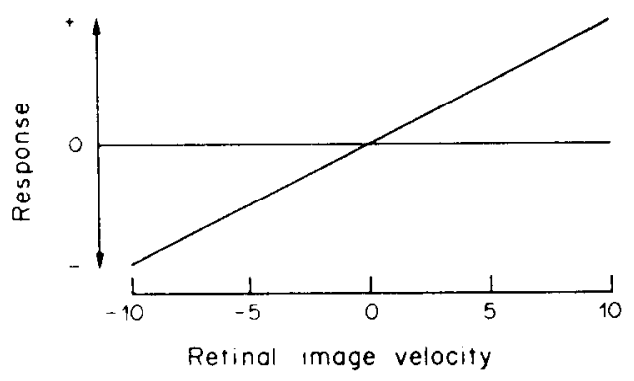

B

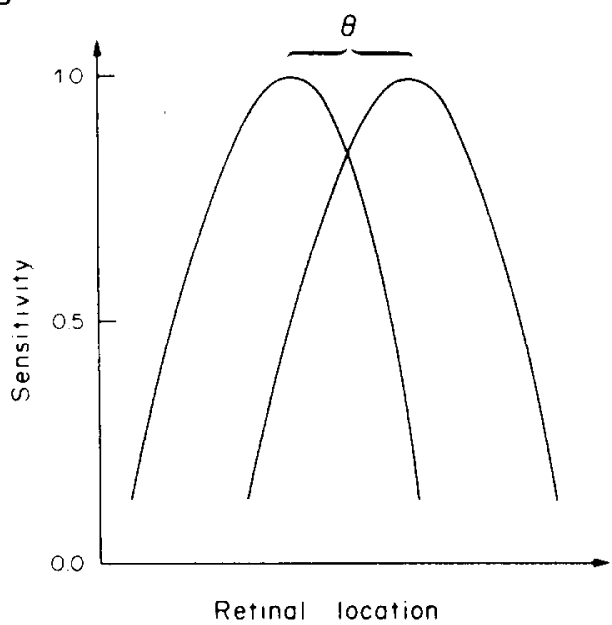

C

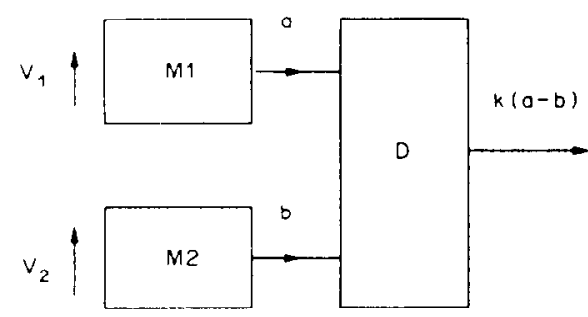

Fig. 2. One-dimensional relative motion element. (A) A hypothetical opponent-motion element's response plotted vs local retinal image velocity. (B) Sensitivity profiles for two opponent-motion elements that are driven from different retinal locations. The difference between their outputs gives the rate of change of image size independently of motion across the retina. (C) Functional diagram of onedimensional relative motion element. Two opponent-motion elements $\mathrm{M} 1$ and $\mathrm{M} 2$ feed a stage $\mathrm{D}$ whose output is proportional to the difference between the signals ( $a$ and $b$ ) from the opponent-motion elements. This arrangement can be regarded as spatial opponency between two opponentmotion elements. Figure $2(\mathrm{C})$ is modified from Regan $\mathrm{D}$. and Beverley K. I. (1980) J. opt. Soc. Am. 11, 1289-1296.

their sensitivity curves overlap substantially [Fig. 2(B)]. We suggested that these two opponent-motion elements feed a stage (D) which requires two input signals, and whose output is proportional to the difference between these two signals [Fig. 2(C)]. The arrangement shown in Fig. 2(C) can be regarded as a kind of spatial opponency between a pair of opponentmotion elements. Note that Fig. 2(C) shows a functional equivalent of a neural relative motion element; for example, in a physiological element, all the processes shown in Fig. 2(C) might be achieved within a single cell.

In the human visual system the separation $\theta$ between the opponent-motion elements [Fig. 2(B)] can be up to $1.5 \mathrm{deg}$ at least (Beverley and Regan, 1979a), and the sensitivity of a relative motion element falls from maximum to a fraction $1 / e$ in about $1 \mathrm{deg}$ (Beverley and Regan, 1980). Figure 2(B) shows the overlapping sensitivity profiles of two such opponent-motion elements.

Neurons sensitive to relative motion have been found in monkey (Motter and Mountcastle, 1981; Miezen et al., 1982), cat (Bridgeman, 1972; Burns et al., 1972; Regan and Cynader, 1979) and pigeon (Frost and Nakayama, 1983).

Specific sensitivity to dilatation and compression

So far we have discussed one-dimensional relative motion. Now we turn to twodimensional relative motion. When a nonrotating rigid object moves towards the eye, its retinal image grows larger, and when the object moves further away its retinal image grows smaller. This change in retinal image magnification is called "looming". It has long been known that the visual system is sensitive to looming in the sense that changing a stationary object's retinal image size can produce an impression that the object is moving in depth (Wheatstone, 1852). More recently it has been proposed that the visual system contains neural elements whose sensitivity to looming is specific, and that this sensitivity cannot be reduced to classical motion sensitivity (Regan and Beverley, 1978a).

The chief experimental evidence for looming elements is as follows: (1) The adapting effect of a rectangle of constant location and oscillating size is quite different from the adapting effect of the same rectangle when size is held constant while position oscillates. Adapting to oscillating size elevates threshold for oscillating size but has little effect on threshold for oscillating position, while adapting to oscillating position has little effect on threshold for oscillating size (Regan and Beverley, 1978a). The significance of this differential threshold elevation is that the 
retinal image motion of any given edge of the rectangle is identical in the two adapting conditions. Therefore, the differential adapting effect is specific to the relationship between the motion of opposite edges rather than to the motion of any given edge, and this is the basis for suggesting that the site of adaptation is the site of interaction between signals from opposite edges. (2) A second distinction between classical motion adaptation and adaptation to looming is that substantial threshold elevations for looming can be produced by such small oscillations of the adapting rectangle's edges that there is no appreciable effect on sensitivity to translational motion (Regan and Beverley, 1978a). (3) Looming adaptation is almost unaffected by superimposing translational motion on the adapting rectangle's size oscillations even when the translational motion is 8 times faster than the changing-size motion. This finding is the basis for suggesting that visual responses to looming are independent of translational motion (Regan and Beverley, 1980).

\section{Time to collision}

Consider a rectangular target of width $S$ and distance $D$ from the eye that is moving at velocity $V$ at an angle $\beta$ to the line of sight. It has a frontal plane velocity $V \sin \beta$ and a line of sight velocity $V \cos \beta$. The rate of change of angular size of the retinal image $\mathrm{d} \theta / \mathrm{d} t=\left(-S / D^{2}\right) \cdot(\mathrm{d} D / \mathrm{d} t)=(-S V \cos \beta) / D^{2}$. Since $\mathrm{d} \alpha / \mathrm{d} t=\left(\mathrm{d} \alpha_{\mathrm{R}} / \mathrm{d} t\right)-\left(\mathrm{d} \alpha_{\mathrm{L}} / \mathrm{d} t\right)$, where $\left(\mathrm{d} \alpha_{\mathrm{R}} / \mathrm{d} t\right)$ and $\left(\mathrm{d} \alpha_{\mathrm{L}} / \mathrm{d} t\right)$ are, respectively, the angular velocities of the left and right sides of the target's retinal image, then it follows that $\left(\mathrm{d} \alpha_{\mathrm{R}} / \mathrm{d} t\right)-\left(\mathrm{d} \alpha_{\mathrm{L}} / \mathrm{d} t\right)=(S V \cos \beta) / D^{2}$. Thus, an element sensitive to the difference between the velocities of opposite edges of the retinal image responds only to the line of sight component of velocity $V \cos \beta$. It does not respond to the frontal plane component of velocity $(V \sin \beta)$ since $\left[\left(\mathrm{d} \alpha_{\mathrm{R}} / \mathrm{d} t\right)-\left(\mathrm{d} \alpha_{\mathrm{L}} / \mathrm{d} t\right)\right]=0$ for this component. In the special case that $V \sin \beta=0$ (i.e. frontal plane velocity is zero) then, following Hoyle (1960) and Lee (1976), the normalized output of a looming element can directly give time to collision since $T=\theta /\left[\left(\mathrm{d} \alpha_{\mathrm{R}} / \mathrm{d} t\right)-\left(\mathrm{d} \alpha_{\mathrm{L}} / \mathrm{d} t\right)\right]$ where $T$ is time to collision and $\theta$ is the angular separation of the opponent-motion elements in Fig. 2.

\section{Rigid vs nonrigid or rotating objects}

This section discusses evidence that relative motion elements could be involved in the analysis of optic flow patterns. In monocular vision, a change in the size of an object's retinal image is multiply ambiguous. A uniform increase in size could mean either that the object is approaching or that it is growing larger (Piéron, 1931). An increase of retinal image size accompanied by a change of shape could mean that a rigid object is approaching while rotating, or that the object is nonrigid or that a nonrigid object is approaching while deforming. The extent to which the human visual system does resolve these ambiguities, and hypothetical ways in which a resolution might be achieved have been much discussed (Johansson, 1964; Ullman, 1979; Wallach and O'Donnell, 1953; Fieandt and Gibson, 1959).

There is evidence that the human visual system is biased in a way that might provide a stereotyped, though arbitrary, reduction of these ambiguities. After adapting to a rectangle whose size continually increases without changing shape, a subsequently-viewed rectangle appears to be of constant size, but to be moving in depth. However, after adapting to a rectangle whose size increases while the shape changes, a subsequently-viewed rectangle appears to be changing size, but not moving in depth. After a few seconds the aftereffect changes abruptly and the square appears to move in depth at constant size. Either a changing size or a motion-in-depth aftereffect is seen; both are not seen simultaneously (Beverley and Regan, 1979b). This finding can be explained if the visual system is biased so that, providing that the retinal image is of constant shape, the eye responds to a change of retinal image size as though it were caused by the motion in depth of a rigid nonrotating object rather than being caused by an actual change in the object's size. We suppose that the bias fails when the retinal image changes shape while changing size. In evolutionary terms, such a bias would minimize the penalty for nonveridical perception when being approached by a predator (Beverley and Regan, 1979b). Using different arguments, Ullman (1979) has also proposed that the visual system is biased towards motion in depth.

In order to distinguish between, on the one hand, retinal image magnification with no change of shape and, on the other hand, magnification accompanied by shape change, the visual system is presumably required to be sensitive to shape change. One characteristic of the monocular retinal image changes caused by pure motion in depth (looming) that dis- 
tinguishes them from retinal image changes caused by object rotation or object shape change is that, in the case of looming, the rate of change of magnification is the same along all meridia. In principle, this characteristic could be utilized in designing a filter that responded to the motion in depth of a rigid, nonrotating object, but did not respond to rotation or deformation. If such a filter were built up from a number of one-dimensional relative motion elements (Fig. 2), each sensitive to motion along a different meridian, the filter would require to incorporate nonlinear interactions between these responses. In particular, the hypothetical looming filter would respond strongly when: (a) rclative motion elements sensitive to motion along different meridia were activated simultaneously, and (b) the value of $(\mathrm{d} \theta / \mathrm{d} t) / \theta$ was identical for all meridia, where $(\mathrm{d} \theta / \mathrm{d} t) / \theta$ was the object's instantaneous rate of change of angular size and $\theta$ was its instantaneous angular size. There is psychophysical evidence that some such interaction does occur; responses to relative motion along the horizontal direction not only interact with responses to relative motion along the vertical direction, but also obey the $(\mathrm{d} \theta / \mathrm{d} t) / \theta$ requirement (Beverley and Regan, 1980). This evidence indicates that visual responses to two-dimensional changes of retinal image magnification are not the linear sum of responses to one-dimensional changes. We have proposed elsewhere that nonlinear interactions between one-dimensional relative motion elements sensitive to motion along orthogonal directions effectively create larger units that are specifically sensitive to pure looming.

The eye and Vector Analysis

In the notation of Vector Analysis, the instan- taneous retinal velocity field can be mathematically described in terms of the values of $\operatorname{grad} \mathbf{V}, \operatorname{div} \mathbf{V}$ and curl $\mathbf{V}$ at every point in the field (Koenderink and van Doorn, 1976, 1981; Longuet-Higgins and Prazdny, 1980). The following discussion is intended to help readers unfamiliar with Vector Analysis to grasp the physical meaning of div $\mathbf{V}$ and curl $\mathbf{V}$ sufficiently for our immediate purpose. Treatments of Vector Analysis are available at introductory (Schey, 1973) and more rigorous (Rutherford, 1954) levels. Figure 3(A) represents a snapshot of a retinal flow pattern caused by self-motion through the environment. The speed and direction of motion at any point vary over the retinal image, and are indicated by the length and direction of the arrow at that point. The dotted line in Fig. 3(A) encloses an arbitrary area $\Delta a$. Figure 3(B) shows the dotted line and the velocity $V$ at one point on the line. At that point the component of velocity along the line is $V \cos \theta$, and the component of velocity perpendicular to the line is $V \sin \theta$. A small segment of the dotted line, length $\mathrm{d} l$, is shown in black. In this two-dimensional case

$$
\operatorname{div} \mathbf{V}=\operatorname{Lim}_{\Delta a \rightarrow 0} \S \frac{\mathrm{d} l(V \sin \theta)}{\Delta a}
$$

In words, we multiply the small length $\mathrm{d} l$ by the component of $V$ perpendicular to $\mathrm{d} l$ all the way round the dotted line and sum these products. Then we divide by the enclosed area $\Delta a$, and allow $\Delta a$ to tend to zero, to obtain the value of $\operatorname{div} \mathbf{V}$ at one point in the flow field. The value of curl $\mathbf{V}$ in the two-dimensional case is given by

$$
\operatorname{curl} \mathbf{V}=\operatorname{Lim}_{\Delta a \rightarrow 0} \S \frac{\mathrm{d} l(V \cos \theta)}{\Delta a}
$$

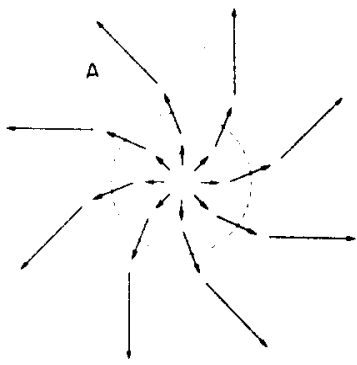

B

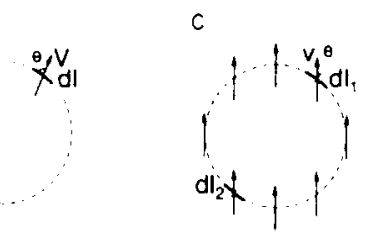

Fig. 3. (A) Illustrates one part of a flow pattern in the retinal image caused by self-motion. The speed and direction of motion at any point vary over the retinal image, and at any point are represented by the length and direction of the arrow at that point. The circular closed line enclosing an arbitrary small area $\Delta a$ is shown again in (B) and (C). (C) The magnitude and direction of velocity $\mathrm{v}$ is the same at every point on the retinal image. Velocity $v$ represents bodily translation of the entire retinal image caused, for example, by moving the point of gaze across the visual field. 
Both $\operatorname{div} \mathbf{V}$ and curl $\mathbf{V}$ can be assigned a value at every point in the flow field. A property of div $V$ and curl $V$ that is of special interest in our present context is that their values are not affected by superimposing translational motion on the visual image as a whole. Thus, their values at any point on the image are not affected by ocular rotation. This can be understood by reference to Fig. 3(C). The translational velocity $v$ is necessarily the same at all points on the image as illustrated. Since, for every small segment $\mathrm{d} l_{1}$ there is a diametrically-opposite segment of equal length $\left(\mathrm{d} l_{2}\right)$, it follows that, in equation (3) above, each $\mathrm{d} l(\sin \theta)$ is cancelled by an equal and opposite contribution so that $\operatorname{div} \mathbf{v}=0$. Similarly, from equation (4), curl $v=0$. In words, the translation velocity $v$ does not contribute to either divergence or curl.

Psychophysical evidence that the human visual system is specifically sensitive to $\operatorname{div} \mathbf{V}$ is described above and evidence for specific sensitivity to rotary motion is described below.

\section{The possible role of div $\mathrm{V}$ elements in the recovery of self-motion}

Translational motion of a nonrotating eye produces a pattern of local deformation and flow in the retinal image that is jointly determined by the eye's motion, and by the threedimensional structure of the environment. In contrast, retinal image motion produced by eye rotation is entirely determined by the rotation independently of the environment (Koenderink and van Doorn, 1976). To describe the retinal image changes caused by a specified motion of the eye through a specified environment of stationary objects is a straightforward geometrical problem that has been worked out in some detail (Gordon, 1965; Koenderink and van Doorn, 1976, 1981; Longuet-Higgins and Prazdny, 1980). The converse problem, however, is little understood at an empirical level; our knowledge of the ways in which the visual system actually does extract self-motion and environmental information from the optic flow pattern is fragmentary. Even at a theoretical level it is not a trivial problem to describe mathematically how this information might, in principle, be recovered. The next paragraphs describe several quite different approaches that have been taken to this converse problem.

One approach has it that the observer compares two or more different views of the scene and first establishes which elements in the several images correspond to a single object in the environment (Ullman, 1979; Marr and Poggio, 1979). A second approach is to assume that the scene is locally rigid and to derive self-motion and the three-dimensional structure of the environment from the flow field (Gibson, 1950; Gordon, 1965; Koenderink and van Doorn, 1976). If the effects of eye rotation can be allowed for or ignored, the observer's self motion and the three-dimensional structure of the external world can, in principle, be recovered from the optic flow pattern (Gibson, 1950; Lee, 1976; Braunstein, 1976; Longuet-Higgins and Prazdny, 1980). In practice, however, it seems that this simplifying assumption is not generally valid: the effects of eye rotation cannot always be ignored (Llewellyn, 1971; Regan and Beverley, 1982). In the special case that parallax information is available, self-motion and evironmental structure can, in principle, be recovered even when the eye rotates while translating (Helmholtz, 1925; Longuet-Higgins and Prazdny, 1980); a directional accuracy of about $0.5 \mathrm{deg}$ can be achieved on the basis of motion parallax (Regan and Beverley, 1984a; Priest and Cutting, 1985).

Longuet-Higgins and Prazdny showed that it is mathematically possible to derive self-motion from the retinal flow field, providing that the local divergence, vorticity and shear of the velocity field are available in addition to the local velocity. As noted above, these spatial derivatives of local velocity have the feature of simple transformation properties with respect to eye rotation (Koenderink and van Doorn, 1976; Longuet-Higgins and Prazdny, 1980), and this could be advantageous in analyzing the flow patterns that contain components due to both eye rotation and eye translation. Psychophysical evidence that the human visual system has specific sensitivities that are roughly equivalent to divergence, vorticity and (possibly) shear detection is described in this paper.

In an experimental study linking looming sensitivity with sensitivity to flow patterns, the flow pattern consisted of 13 bright circular lines, each cut into 48 segments that flowed radially towards or away from the centre. Radial speed was constant over the whole stimulus. After adapting to this flow pattern, sensitivity to changes in the size of a small $(0.5 \mathrm{deg})$ square was depressed when the test square was near the former location of the flow pattern's focus. but changing-size sensitivity was not affected elsewhere (Regan and Beverley, 1979b; Regan, Beverley and Cynader, 1979). Sensitivity to 


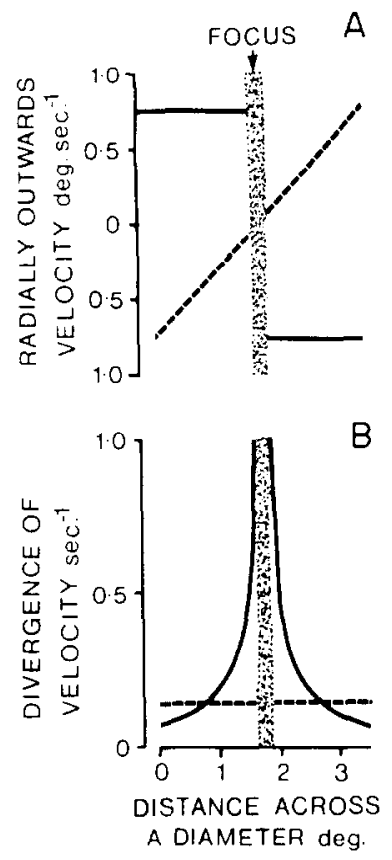

Fig. 4. (A) The continuous line plots radial velocity across a diameter of the flow pattern used experimentally. The hatched area at the centre represents a black disk that occluded the centre of the pattern. The broken line plots radial velocity for the different kind of flow pattern that would be produced by a zooming lens. (B) The continuous line plots the distribution of $\operatorname{div} V$ across a diameter of the flow pattern used experimentally, and corresponds to the continuous line in (A). The broken line plots the distribution of $\operatorname{div} \mathbf{V}$ for the zooming-type flow pattern represented by the broken line in $\mathbf{A}$.

translational motion was not affected. This finding can be re-analyzed in terms of visual sensitivity to div V. Figure 4(A) shows the pattern of instantaneous local velocity (continuous line). The associated $\operatorname{div} \mathbf{V}$ has been calculated in Fig. 4(B) (continuous line). Div $\mathrm{V}$ was large at the centre of the pattern coincident with the focus of flow, and small everywhere else. In such a radially-expanding target, sensitivity to changes in local $\operatorname{div} \mathbf{V}$ might also be involved in superthreshold velocity discrimination. It is of interest that superthreshold velocity discrimination with this target correlated with performance in high-speed, visually-guided, low-level flight both with real jet aircraft and in a flight simulator (Kruk and Regan, 1983; Kruk et al., 1983).

The dashed line in Fig. 4(A) illustrates the instantaneous pattern of local velocity that would be produced by a zooming lens and Fig. 4(B) (dashed line) shows this pattern has no maximum of $\operatorname{div} \mathbf{V}$ at the focus. Experimentally, subjects were unable to locate the focus of expansion in a one-dimensional version of this flow pattern, but when a local maximum of div $\mathbf{V}$ was introduced, localization accuracy improved substantially (Regan and Beverley, 1982), consistent with the idea that the visual system is sensitive to local div V. An observer's destination, however, does not coincide with a local maximum of $\operatorname{div} \mathbf{V}$ in all environmental geometries; neither sensitivity to $\operatorname{div} \mathbf{V}$ nor sensitivity to a flow pattern's focus provide a general explanation for the visual guidance of self-motion (Regan and Beverley, 1982, 1984a; Regan, 1985). Possible alternative explanations include an eye movement strategy based on a template-matching procedure (Regan, 1985).

\section{SECIION 2}

Discriminating the directions of motion in depth

The direction of a target's motion in depth can be discriminated with a remarkable acuity of about $0.2 \mathrm{deg}$ when viewing is binocular (Beverley and Regan, 1975). It has been proposed that this high acuity can be explained in terms of sensitivity to relative motion, in this case a velocity ratio (Beverley and Regan, 1973). Because the two eyes are a few $\mathrm{cm}$ apart, the left and right eyes' images of an object moving in depth move with different velocities $\left[V_{\mathrm{S}}\right.$ and $V_{\mathrm{D}}$ respectively, see Fig. 1(D)]. The ratio $V_{\mathrm{D}} / V_{\mathrm{S}}$ is uniquely related to the direction of motion in depth. Figure 5 shows psychophysical evidence that the human visual system contains elements tuned to the velocity ratio. In the experiment of Fig. 5 the subject viewed two dot patterns, one with each eye. Each pattern oscillated from side to side at the same rate, but with different velocities. The subject's task was to set thresholds for just-visible motion in depth. Figure 5 shows threshold elevations caused by separately adapting to four different $V_{\mathrm{D}} / V_{\mathrm{S}}$ ratios.

These data can be understood if the visual system contains eight kinds of binocular element, cach tuned to a different value of $V_{\mathrm{D}} / V_{\mathrm{S}}$. four preferring movement towards the head and four preferring movement away from the head. For the purpose of the following discussion, note that adapting to a direction inclined just to the left of the nose (open circles) gives a clearly different threshold elevation curve than adapting to a direction inclined just to the right of the nose (crosses), consistent with the idea that the two central elements sharply differentiate between trajectories to the left and right of the nose. By analogy with Hering's theory of colour vision it has been suggested that, in binocular 


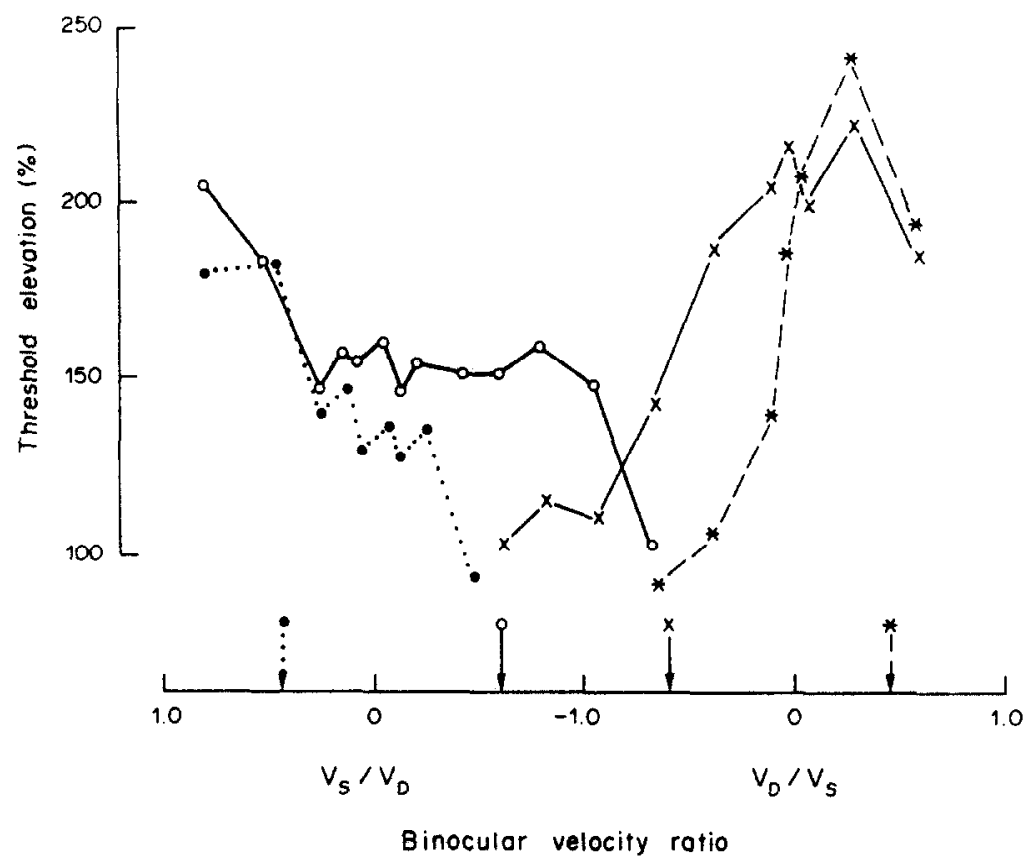

Fig. 5. Thresholds for the detection of motion in depth were measured for different ratios of the left $\left(V_{D}\right)$ and right eye's $\left(V_{\mathrm{S}}\right)$ image velocities. Threshold elevations were plotted as ordinates versus the velocity ratio of the test oscillation after adapting to four different directions of motion in depth. A negative sign means that $V_{S}$ and $V_{D}$ are in opposite directions. Solid circles, fine continuous line-adapting ratios $V_{\mathrm{S}} / V_{\mathrm{D}}=+0.5$ (trajectory to left of left eye). Open circles, heavy continuous line-adapting ratio $V_{\mathrm{S}} / V_{\mathrm{D}}=+0.5$ (trajectory passes between eyes to left of centre). Crosses, fine continuous line-adapting ratio $V_{\mathrm{D}} / V_{\mathrm{S}}=-0.5$ (trajectory passes between eyes to right of centre). Stars, broken line-adapting ratio

$V_{\mathrm{D}} / V_{\mathrm{S}}=+0.5$ (trajectory passes to right of right eye). Arrows mark the adpating stimulus ratios.

vision, directional discrimination is mediated by interaction between these overlapping elements, much as colour discrimination is mediated by opponency between the three colour mechanisms. Evidence supporting this idea is that directional discrimination is most accurate (about $0.2 \mathrm{deg}$ ) for trajectories near the crossover point of the binocular elements (Beverley and Regan, 1975; Regan, 1982).

Monocular discrimination of the direction of motion in depth is a different problem, but can be approached analogously to the binocular case. An object moving along an arbitrary trajectory is simultaneously looming and translating. Figure 6 illustrates how the ratio between the velocities of a square's vertical edges is related to its direction of motion. When the centre of the square moves directly through the eye, the speeds of the left and right edges are equal and opposite $\left[V_{\mathrm{L}} / V_{\mathrm{R}}=-1.0\right.$ in Fig. 6(A) $]$. When the square translates to the right as it comes towards the eye but still hits the eye, the left and right edges move in opposite directions with the left edge moving slower than the right $\left[V_{L} / V_{R}=-0.5\right.$ in Fig. 6(B)]. When the square's left edge just grazes the eye, the left edge appears stationary $\left[V_{L} / V_{R}=0\right.$ in Fig. $\left.6(\mathrm{C})\right]$. When the square passes to the right of the eye, the left and right edges move in the same direction, the left edge slower than the right $\left[V_{L} / V_{R}=+0.5\right.$ in Fig. 6(D)], and when the square translates in the frontal plane, left and right edges move identically $\left[V_{L} / V_{R}=+1.0\right.$ in Fig. $\left.6(\mathrm{E})\right]$. Subjects are quite sensitive to differences in the $V_{L} / V_{R}$; the trajectory $V_{\mathrm{L}} / V_{\mathrm{R}}=1.1$ is seen to be clearly tilted in depth compared with $V_{\mathrm{L}} / V_{\mathrm{R}}=1.0$.

One possible explanation for monocular discrimination of the direction of motion in depth would be that the visual pathway contains several elements tuned either to different $V_{L} / V_{R}$ ratios or to different combinations of changing size and frontal plane motion. For example, one kind of element might prefer increasing size combined with rightward motion (stimulus A), while a second kind preferred increasing size combined with leftward motion (stimulus B). Discrimination would be determined by the relative activity of these notional elements. In order to test for the presence of such selective sensitivities, a monocular adaptation experi- 
A
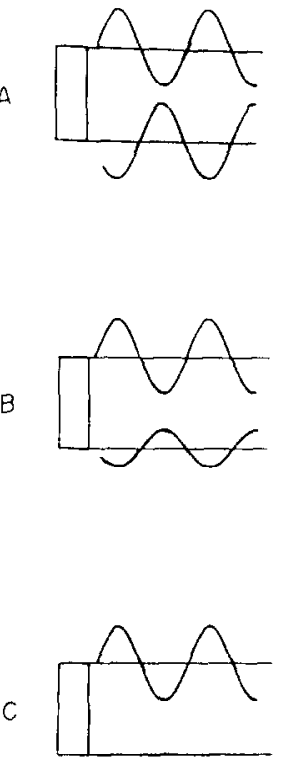

D
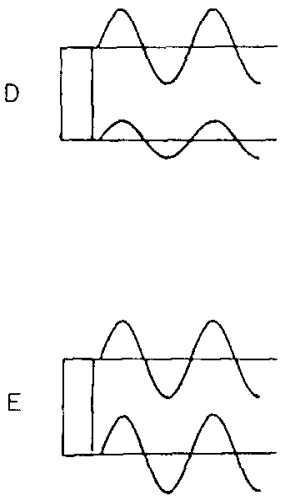
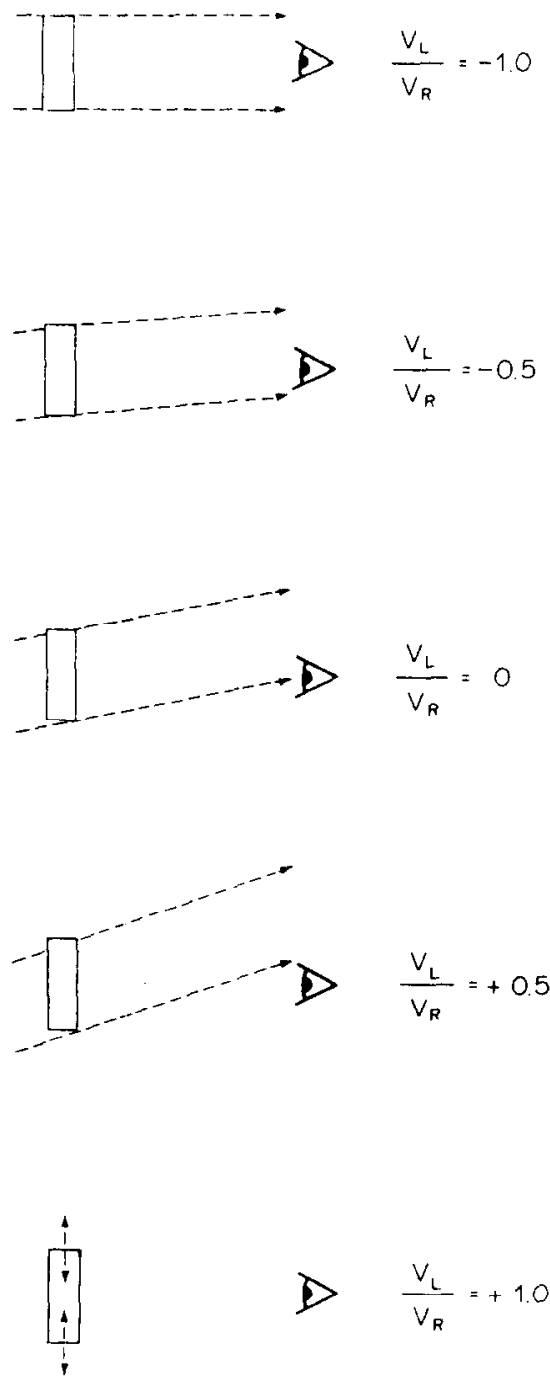

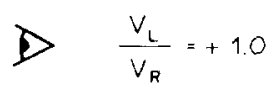

Fig. 6. Monocular correlate of the direction of motion in depth. (A-E) A square object oscillating along various directions of motion in depth with respect to the eye. (F-J) The oscillations of the object's left and right edges seen by the eye. $V_{L}$ and $V_{R}$ are the instantaneous angular velocities of the left and right edges. A negative sign means that $V_{\mathrm{L}}$ and $V_{\mathrm{R}}$ are in opposite directions. When $0<\left(V_{\mathrm{L}} / V_{\mathrm{R}}\right)<1.0$, the square would pass to the right of the eye. When $\left(V_{L} / V_{R}\right)=0$ the left edge of the square would just graze the eye. When $-1.0<\left(V_{\mathrm{L}} \cdot V_{\mathrm{R}}\right)<0$ the square would hit the eye. Similar relations hold for $\left(V_{\mathrm{R}} / V_{1}\right)$.

ment was carried out whose rationale was analogous to the binocular experiments of Fig. 5. In the monocular experiment, subjects set oscillation thresholds for stimuli $\mathrm{A}$ and $\mathrm{B}$ before and after adapting to stimulus $A$, and before and after adapting to stimulus B. Figure 7 plots postadaptation threshold elevations vs the $V_{L} / V_{\mathrm{R}}$ ratios of the 12 different test stimuli. Test and adapting squares were centrally viewed, $1.0 \mathrm{deg}$ side length and of luminance $12 \mathrm{~cd} / \mathrm{m}^{2}$ superimposed on a $10 \times 10$ deg background of luminance $25 \mathrm{~cd} / \mathrm{m}^{2}$. Each edge oscillated sinusoidally with a frequency of $1.0 \mathrm{~Hz}$. The initial adaptation period was $15 \mathrm{~min}$. The trial interval was $6 \mathrm{sec}$ with $20 \mathrm{sec}$ readapt between trials. Four different adapting stimuli were used: L12R6 inphase (solid circles, fine dotted line); L12R6 antiphase (open circles, heavy continuous line); L6R 12 antiphase (crosses, fine continuous line); L6R 12 inphase (stars, broken line), where $L$ and $R$ refer to the left and right edges and the numbers are oscillation amplitudes in min arc.

Figure 7 disproves the idea that threshold elevations were entirely determined by the oscillations of individual edges. For example, the two L12R6 adapting stimuli had identical oscillation amplitudes and velocities, but gave quite 


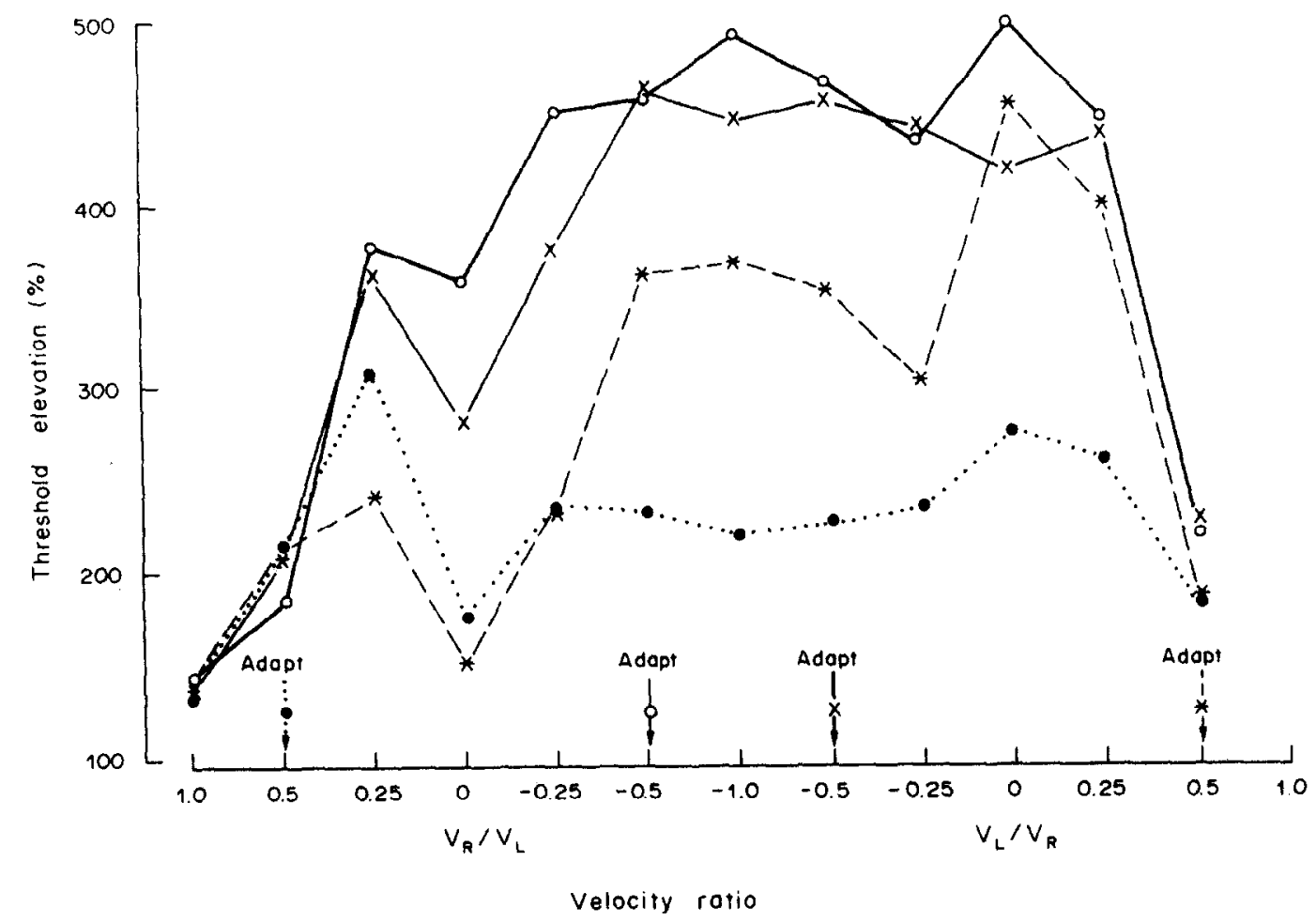

Fig. 7. Monocular threshold elevations caused by adapting to different directions of motion in depth. Abscissae plot ratios between the velocities of the test square's left and right edges (see Fig. 7). The four curves are for the four adapting directions arrowed. Solid circles, fine dotted line-adapting ratio $V_{R} / V_{L}=+0.5$ (trajectory to left of eye). Open circles, heavy continuous line-adapting ratio $V_{R} / V_{L}=-0.5$ (trajectory passes through eye just left of centre). Crosses, fine continuous line-adapting ratio $V_{L} / V_{R}=-0.5$ (trajectory passes through eye just right of centre). Stars, broken line--adapting ratio $V_{\mathrm{L}} / V_{\mathrm{R}}=+0.5$ (trajectory to right of eye).

different threshold elevations (compare solid and open circles). These two adapting stimuli differed only in the phase relation between opposite edges. On the other hand, the Fig. 7 monocular data differ from the Fig. 5 binocular data in that adapting to trajectories inclined just to the left and right of centre did not produce clearly different elevation curves (compare open circles and crosses in Figs 5 and 7) so that, in contrast with the binocular findings, there was no evidence for elements that sharply distinguished between trajectories inclined slightly to the left and right of a collision course. The only evidence for a monocular element that preferred increasing size with rightward motion and decreasing size with leftward motion was the asymmetry of the Fig. 7 curve marked by stars. There was no evidence for elements tuned to the converse $V_{\mathrm{L}} / V_{\mathrm{R}}$ ratio (solid circles). Thus, the Fig. 7 data can almost entirely be explained by assuming that, in contrast with the binocular analysis of motion in depth, monocular analysis is chiefly into orthogonal velocity components.
These components comprise motion towards and away from the eye along the line of sight, and components parallel to the frontal plane. [Different directions in the frontal plane would be dealt with by different frontal plane motion elements as described by Sekuler, Pantle and Levinson (1978).] On the other hand, Fig. 7 gives some suggestion that, in addition, there might be elements tuned to values of $V_{\mathrm{L}} / V_{\mathrm{R}}$ other than +1.0 and -1.0 .

\section{The speed of motion in depth}

For an object of given size and viewing distance the object's speed of motion in depth determines the instantaneous rate of change of binocular disparity and also the instantaneous rate of change of size of its retinal images. Each of these two physical relationships has its separate subjective counterpart. Changing binocular disparity with no change in size can create the impression that the object is moving in depth even though it is not; changing the size of an object's retinal images can create the impression 
that the object is moving in depth, even though it is not.

An object's speed of motion in depth cannot be obtained from the rate of change of size unless the object's distance is available. Nevertheless, a monocularly-viewed changing-size square appears to be moving at some definite speed even when no visual reference marks are visible. For superthreshold speeds of motion in depth, the initial speed of the motion-in-depth aftereffect is approximately proportional to the adapting rate of change of size both for motion towards and for motion away from the head. Experimentally, thresholds for motion-in depthsensation are approximately the same whether produced by increasing or by decreasing size (Regan and Beverley, 1978b).

Turning to motion in depth produced stereoscopically, there are several possible ways in which the visual system might compute the speed of motion in depth. For example, the speed of motion in depth can be obtained from the rate of change of binocular disparity. We suppose that this would be obtained by first obtaining monocular velocities $V_{\mathrm{D}}$ and $V_{\mathrm{S}}$ [Fig. 1(D)], and then computing the binocular difference $\left(V_{\mathrm{D}}-V_{\mathrm{S}}\right)$. We can reject the alternative procedure of first obtaining static binocular disparity and then computing its rate of change, because some subjects have areas of the visual field that are "blind" to static disparity, yet have normal sensitivity to motion in depth (Richards and Regan, 1973; Regan et al., 1985b). Rather than by computing binocular velocity difference (i.e. rate of change of disparity), an alternative method of obtaining the speed of motion in depth is from the hypothetical ratio-tuned neural elements described in the preceding section. Suppose that the ensemble of elements that respond best to some given $V_{\mathrm{D}} / V_{\mathrm{S}}$ ratio [Fig. 1(D)] comprise elements with low, intermediate and high velocity thresholds. Low speeds of motion in depth would excite only lowthreshold elements, while high speeds would excite all the elements that respond best to the particular $V_{\mathrm{D}} / V_{\mathrm{S}}$ ratio. The speed of motion in depth would be uniquely represented by the total number of active elements, and the direction of motion in depth would be represented by the balance of activity across elements tuned to different $V_{\mathrm{D}} / V_{\mathrm{S}}$ ratios. Currently available experimental evidence is not sufficient to decide whether visual responses to the speed of motion in depth can be entirely accounted for in terms of an ensemble of $V_{\mathrm{D}} / V_{\mathrm{S}}$ ratio-tuned elements, or whether additional elements must be postulated, for example elements sensitive to $\left(V_{\mathrm{D}}-V_{\mathrm{S}}\right)$, the rate of change of disparity.

The movement-in-depth signals caused by changing size and by changing disparity are identical in the sense that the impression of motion in depth can be cancelled when the two stimuli are pitted against each other (Regan and Beverley, 1979a). If we suppose that changingsize signals and changing disparity signals converge before motion-in-depth signals are generated, then we can compare the strengths of changing-size and changing-disparity signals by cancellation. Measurements were made of the rate of change of size $\left(\theta_{S} \mathrm{deg} / \mathrm{sec}\right)$ required to cancel the impression of motion in depth produced by different rates of change of disparity $\left(\theta_{\mathrm{D}} \mathrm{deg} / \mathrm{sec}\right)$. The plots were linear on $\log -\log$ axes, so that $\theta_{\mathrm{D}}=k\left(\hat{\theta}_{\mathrm{S}}\right)^{n}$. The value of $\mathrm{n}$ was less than $1.0(0.28,0.34$ and 0.56 for 3 subjects $)$, suggesting that the changing disparity grows relatively more effective as a stimulus for motion in depth as line of sight speed increases. These data for unidirectional motion in depth should be compared with data for a target that oscillates in depth; changing disparity is relatively more effective than changing size at low frequencies, and sensitivity to both stimuli falls off similarly above $1 \mathrm{~Hz}$.

It may be that the peripheral relative motion elements illustrated in Fig. 2(C) are shared by the looming and stereoscopic motion systems. Suppose that the subject views a real moving object and a stationary reference point whose

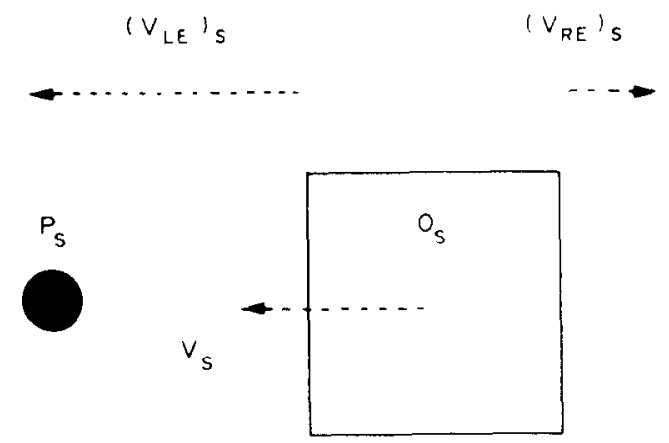

Fig. 8. $P_{s}$ and $O$ sespectively mark the left eyc's retinal images of a stationary reference point and an object that is moving in depth relative to the reference. Reginal image motion includes both changing size and stereoscopic correlates of the object's motion in depth. The left edge of $O$ has velocity $\left(v_{L t}\right)_{S}$ and the right edge $\left(v_{K I}\right)_{S}$. both with respect to $P$. The changing size component is given by $\left[\left(v_{R E}\right)_{S}-\left(v_{L E}\right)_{S}\right]$, and the left eye's stereoscopic component is $\left.V_{\mathrm{S}}=1 / 2\left[\left(t_{\mathrm{R}: \mathrm{t}}\right)+\left(v_{\mathrm{L}, \mathrm{t}}\right)_{\mathrm{S}}\right)\right]$ 
retinal images are in the left eye are marked respectively $O_{\mathrm{s}}$ and $P_{\mathrm{s}}$ in Fig. 8. Relative to $P_{\mathrm{s}}$, $O_{\mathrm{s}}$ has a changing-size component of motion plus a stereoscopic component of motion $\left(V_{\mathrm{s}}\right)$. We suppose that one relative motion element receives inputs from $P$ and from the left edge of $O$, and signals the value of $\left(v_{\mathrm{LE}}\right)_{\mathrm{s}}$, while a second relative motion element receives inputs from $P$ and from the right edge of $O$, and signals the value of $\left(v_{\mathrm{RE}}\right)_{\mathrm{s}}$. The stereoscopic component of motion for the left eye could be obtained by computing $V_{\mathrm{S}}=1 / 2\left[\left(v_{\mathrm{RE}}\right)_{\mathrm{S}}-\left(v_{\mathrm{LE}}\right)_{\mathrm{S}}\right]$, thus eliminating the changing-size component of motion. The value of the stereoscopic component for the right eye $\left(V_{\mathrm{D}}\right)$ could be obtained similarly. As discussed earlier, the relative activity within an ensemble of binocularly-driven elements sensitive to different values of the ratio $\left(V_{\mathrm{S}} / V_{\mathrm{D}}\right)$ could indicate both the direction and speed of motion in depth or, alternatively, speed could be obtained by computing $\left(V_{\mathrm{D}}-V_{\mathrm{S}}\right)$. This model accounts for the observation that stereoscopic motion in depth is not seen when there is no reference mark in the visual field (Erkelens and Collewijn, 1985), because indeterminate outputs are then obtained from the relative motion elements that signal $v_{\mathrm{LE}}$ and $v_{\mathrm{RE}}$.

Relative motion elements driven by the left and right edges of object $O$ (Fig. 8) give outputs even when no reference mark is visible in the visual field. This explains our finding that a single target viewed against a featureless background appears to move in depth when its size is changed, even though stereoscopic motion-indepth stimulation is comparatively ineffective (Regan et al., 1985, 1985a).

We have not discussed vergence eye movements in the context of motion-in-depth perception. This is because vergence eye movements as fast as $30 \mathrm{deg} / \mathrm{sec}$ do not in themselves generate any impression of motion in depth. Furthermore, motion-in-depth perception generated by changing disparity is not affected by simultaneous changes in ocular vergence. For example, in one experiment motion-in-depth thresholds were measured for a $0.25 \mathrm{~Hz}$ oscillation of relative disparity in the two conditions that $0.25 \mathrm{~Hz}$ vergence oscillations occurred (a) inphase, and (b) in antiphase with the disparity oscillations. Thresholds were the same in the two conditions for all three subjects tested even when the speed and amplitude of vergence changes were more than 50 times larger than the disparity thresholds (Regan et al., 1985, 1985a).

\section{SECTION 3}

\section{Visual sensitivity to shearing motion}

Relative motion for which velocity varies perpendicularly to the direction of motion is called shearing motion [Fig. 1(B)]. Nakayama and Tyler (1981) studied shearing motion thresholds as a function of spatial frequency. Each of the dots in their random dot target oscillated at the same rate along a horizontal direction, and the amplitude of oscillation varied sinusoidally in the vertical direction. In the spatial frequency domain, oscillatory motion thresholds fell as grating frequency was reduced, reaching a shallow minimum at about $0.1-0.6 \mathrm{c} / \mathrm{deg}$. In the time domain, oscillatory thresholds fell as temporal frequency was progressively reduced, reaching a minimum at $2 \mathrm{~Hz}$ (Golomb et al., 1985). Figure 9(A) illustrates a bar whose boundaries were defined entirely by shearing motion. Figure 10(A) and (B) shows visual detection thresholds for this bar. These data are the spatiotemporal transient equivalent of oscillatory motion thresholds for shearing motion using a sinewave grating.

Now we turn to the question whether visual responses to shearing motion [Fig. 9(A)] differ from visual responses to compressional motion, i.e. velocity differences perpendicular to the boundary [Fig. 9(B)]. In Fig. 10, thresholds were similar for shearing motion (triangular symbols) and for compressional motion (square symbols); this similarly held over the entire range of bar widths [Fig. 10(A)], and presentation times [Fig. 10(B)]. On the other hand, Richards and Liberman (1982) found that sensitivity to shearing and compressional boundaries differ in some subjects, especially at peripheral locations in the visual field. Again, MacLeod et al. (1983) found that sensitivity to compressional wave motion is preserved up to a spatial frequency $2-3$ times higher than for shearing wave motion. In order to resolve this disagreement we searched for differences between visual responses to edges defined by these two kinds of relative motion by carrying out a cross-adaptation experiment.

The experiment was to measure detection threshold elevations for a Fig. 9(B) bar after adapting to a Fig. 9(B) bar [condition $\left(\mathrm{TE}_{\mathrm{P}}\right)_{\mathrm{P}}$ ] and after adapting to a Fig. 9(A) bar [condition $\left(T_{\mathrm{p}}\right)_{\mathrm{S}}$. Then postadaptation detection threshold elevations were measured using as the test a Fig. 9(A) bar [conditions $\left(\mathrm{TE}_{\mathrm{S}}\right)_{\mathrm{p}}$ and $\left(\mathrm{TE}_{\mathrm{S}}\right)_{\mathrm{S}}$ ]. Our rationale was that a finding that $\left(T E_{\mathrm{P}}\right)_{\mathrm{P}}>\left(\mathrm{TE}_{\mathrm{P}}\right)_{\mathrm{S}}$ and $\left(\mathrm{TE}_{\mathrm{S}}\right)_{\mathrm{S}}>\left(\mathrm{TE}_{\mathrm{S}}\right)_{\mathrm{p}}$ would have 


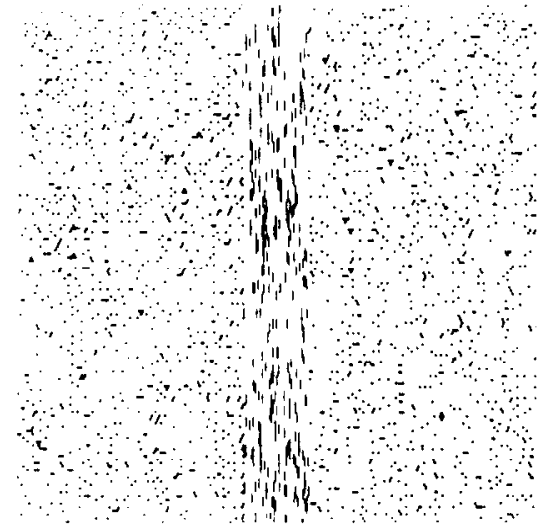

(A)

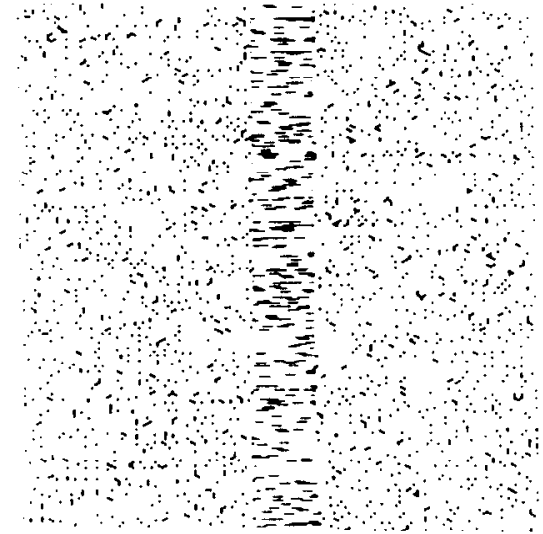

(B)

Fig. 9. (A) The vertical bar's boundaries are almost entirely defined by relative motion parallel to the boundaries ( $\mathrm{S}$ or shearing houndaries). (B) The vertical bar's boundaries are almost entirely defined by relative motion perpendicular to the boundaries ( $P$ or perpendicular motion boundaries). The visual display was photographed so that moving dots are represented as short lines.

provided evidence that the perpendicular motion $(\mathrm{P})$ boundaries and shearing motion $(\mathrm{S})$ boundaries are processed differently.

In attempting this experiment, we encountered difficulty in measuring thresholds; even when no clear boundary was visible, subjects could infer the presence of the bar by observing relative motion between areas of the dot pattern. For example, there was sometimes an impression of global rotary motion. In order to unconfound detection of motion from detection of the bar we used a technique previously developed to study binocular vision (Regan and Beverley, 1973).

Adapting and test patterns were a $4 \mathrm{deg}$

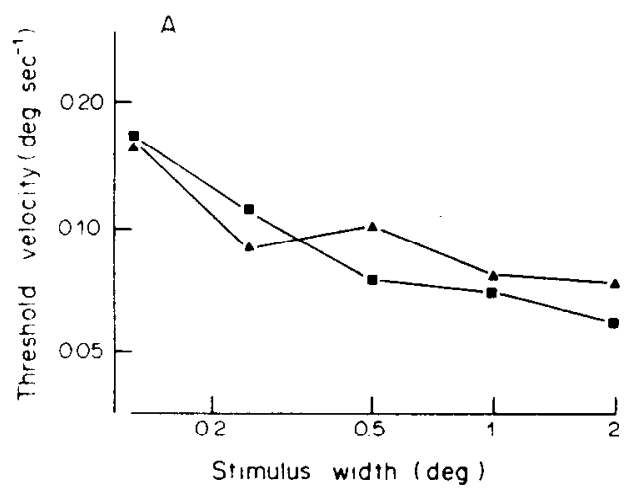

square array of random dots superimposed on a uniformly illuminated background subtending $11 \mathrm{deg}$ (vertical) $\times 15 \mathrm{deg}$. During the adaptation phase all dots executed $0.5 \mathrm{~Hz}$ triangular wave oscillations with the same amplitude. Within the bar the phase was opposite to the phase outside. This gave a strong adapting stimulus as the bar was continuously visible. During the test intervals, all dots oscillated sinusoidally with the same amplitude, either vertically [Fig. 11(A)] or horizontally [Fig. 11(B)]. The test stimulus differed from the adapting stimulus because the oscillation frequency within the vertical bar-shaped area was different from the frequency in surrounding

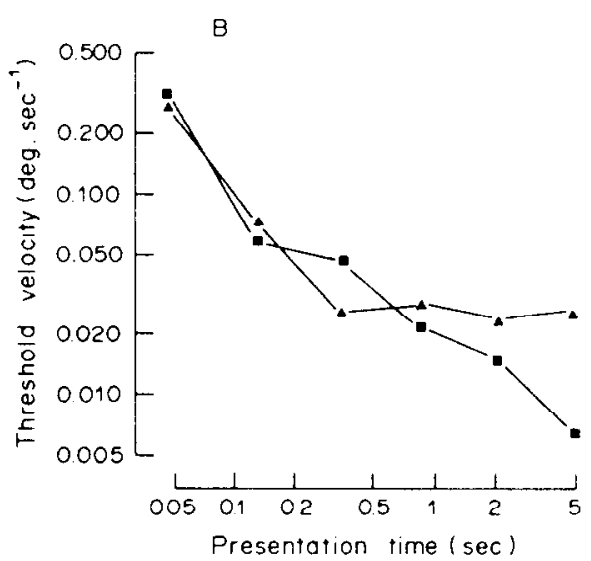

Fig. 10. Detection thresholds for bars whose boundaries were defined by relative motion parallel to the boundaries, i.e. shearing motion (open and solid triangles) and for bars whose boundaries were defined by relative motion perpendicular to the boundaries (open and solid squares). (A) Abscissae plot bar widths. The bar's length was always $8 \mathrm{deg}$ and presentation time $150 \mathrm{msec}$. (B) Abscissae plot presentation time. The bar's length was $8 \mathrm{deg}$ and the width was $1.0 \mathrm{deg}$. Dots subtended $1 \mathrm{~min}$ arc and were $1.5 \mathrm{log}$ units above detection threshold. There were 40 dots per $\mathrm{deg}^{2}$, the whole dot pattern subtended 8 deg square and was superimposed on a homogeneous background $18 \mathrm{deg}$ square of luminance $34 \mathrm{~cd} / \mathrm{m}^{2}$. 
A

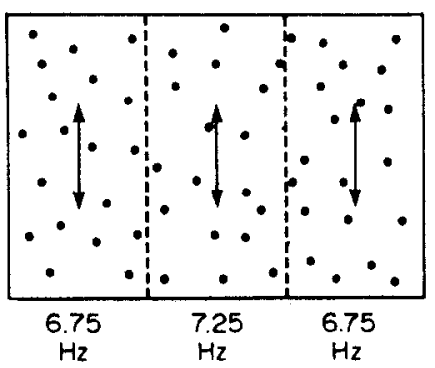

$B$

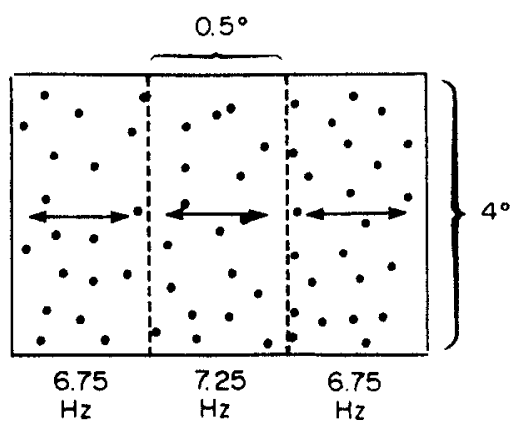

ADAPT

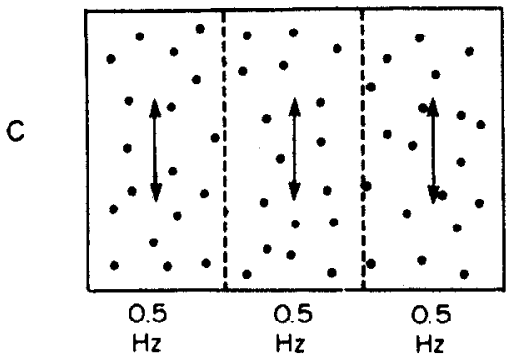

D

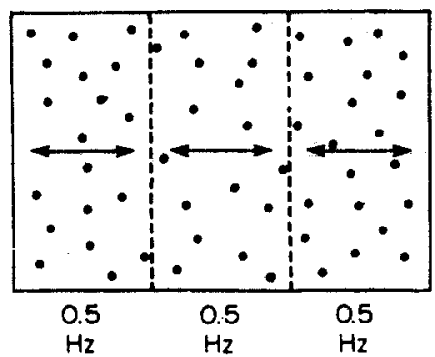

Fig. 11. The two test stimuli are shown in (A) and (B). All dots oscillated with the same amplitude, but within a central bar the frequency $(7.25 \mathrm{~Hz})$ was different to the frequency $(6.75 \mathrm{~Hz})$ in the surround. Relative motion was paraliel to the bar's major boundaries in (C) and perpendicular in (D). (C, D) The two adapting stimuli. All dots oscillated at the same $0.5 \mathrm{~Hz}$ frequency and with the same amplitude, but within a central bar oscillations were in antiphase with the surround. Relative motion was parallel to the bar's major boundaries in (A) and perpendicular in (B).

areas. Dots oscillated at $F=6.75 \mathrm{~Hz}$ outside the bar and at $F+\Delta F=7.25 \mathrm{~Hz}$ outside. Neither one of these frequencies was related to the $\Delta F=0.5 \mathrm{~Hz}$ frequency at which the visibility of the edges changed and the bar appeared and disappeared; $\Delta F$ could be manipulated independently of $F$, and $F$ could be manipulated independently of $\Delta F$, thus enabling the effect of dot oscillation frequency to be dissociated from the oscillation frequency of the figure-ground or boundary processing. Our rationale for this "beat frequency" technique was that the $0.5 \mathrm{~Hz}$ variations in figure-ground segregation would help to define the subject's criterion by providing a clear distinction between the presence of the bar figure and the motion of the dots. Our further intent was to selectively reduce visual ability to segregate figure from ground while having minimal adapting effect on classical sensitivity to motion. The adapting stimulus adapted both figure-ground and dot motion mechanisms to a frequency of $0.5 \mathrm{~Hz}$, but the test stimulus frequency was $0.5 \mathrm{~Hz}$ only for figure-ground; dot motion was at a much higher frequency.

Initial adaptation was for $5 \mathrm{~min}$, and the method of adjustment was used with $6 \mathrm{sec}$ test periods and $30 \mathrm{sec}$ readapt between test periods. Baselines were measured after adapting to a stationary dot pattern.

Although we found threshold elevations to be small in all conditions ( $44 \%$ postadaptation change at most), thresholds for detecting an $\mathrm{S}$ bar and a $\mathrm{T}$ bar seemed to be differently affected 
A

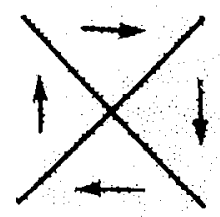

B

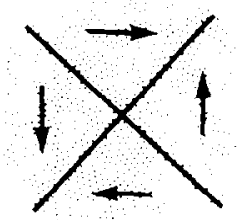

C

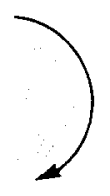

Fig. 12. Rotary motion. Adapting stimuli (A and B) and test stimulus (C). In both (A) and (B) every dot oscillated sinusoidally along a straight line. Every dot had the same amplitude and frequency of oscillation. In (A) and (B) the stimulus areas were divided into four quadrants. The arrows show how the relative phases of oscillation were arranged so that (A) had a component or rotation about the centre, but (B) had not. Test stimulus (C) was an area of dots that rotated sinusoidally to and fro about its centre.

by adapting to an $\mathrm{S}$ bar and a $\mathrm{T}$ bar, consistent with the idea that $\mathrm{S}$ and $\mathrm{T}$ boundaries are processed differently. In three separate experiments the values of $\left(\mathrm{TE}_{\mathrm{S}}\right)_{\mathrm{S}} /\left(\mathrm{TE}_{\mathrm{T}}\right)_{\mathrm{S}}$ were $3.1,2.2$ and 2.8. Adapting to a $\mathrm{T}$ bar produced elevations comparable to the noise level.

\section{SECTION 4}

\section{Visual sensitivity to rotary motion}

It has been suggested that the visual pathway might contain elements that are specifically sensitive to rotary motion, and are distinct from elements sensitive to linear motion (LonguetHiggins and Prazdny, 1980). One problem in testing this suggestion is that rotary motion can be approximated by many short linear trajectories, so that a rotary stimulus will stimulate many visual fields sensitive to linear motion. In an attempt to circumvent this problem, threshold elevations caused by two adapting stimuli were compared (Regan and Beverley, 1985). One had a rotary component of motion, but the other did not. The test stimulus was pure rotary motion. Figure 12 illustrates the rationale. A 2 deg diameter circular area of random dots was divided into four quandrants. The dots in any given quadrant oscillated sinusoidally along a straight line, all with the same peak to peak amplitude. The amplitude and frequency of oscillation were the same in Fig. 12(A) and (B). The only difference between adapting stimuli $12 \mathrm{~A}$ and $\mathrm{B}$ was in the relative phasing of different quadrants. In Fig. 12(A), all the dots moved clockwise or counterclockwise together so that the circular disc had a strong rotary component of motion about the centre. In 12(B), the relative phasing of the four quadrants was as shown by the arrows, giving the circular disc zero net rotary component of oscillation about the centre. Although the adapting stimu- lus had a net rotary component in A but not in $\mathrm{B}$, the linear oscillations within any given quadrant were the same in $\mathrm{A}$ and $\mathrm{B}$; both adapting stimuli consisted of four linear oscillations, and nothing else. The $1 \mathrm{deg}$ diameter test stimulus [Fig. 12(C)] was pure rotary motion. Subjects adjusted the amplitude of oscillation until rotary oscillation of the whole area was just visible. Adapting stimulus $\mathrm{A}$ produced greater threshold elevations than adapting stimulus B, consistent with the idea that responses to rotary motion cannot entirely be explained in terms of responses to linear motion. It is unlikely that the different effects of the two adapting stimuli can be attributed to the finite receptive field size of linear motion elements since this implies that, after adapting to stimulus B (Fig. 12), motion sensitivity would be higher along the quadrant boundaries than within the quadrants. There was no evidence of this inhomogeneity in the subsequently-presented test stimulus C (Fig. 12).

Julesz and Hesse (1970) found that regions of a textured pattern composed of thousands of small elements could not easily be discriminated solely on the basis of differences in the direction of rotation of individual small elements. There is, however, an important difference between the pattern used by Julesz and Hesse and the patterns used in the present study: the Fig. 12(A) and $(C)$ patterns rotated bodily about a single axis while, in Julesz and Hesse's pattern, each of the many small elements rotated about its own geometric centre. Julesz and Hesse's finding makes it unlikely that the visual system contains detectors with small receptive fields that respond specifically to the mathematical quantity curl $\mathbf{V}$, but this does not conflict with our proposal that the visual system contains elements that are sensitive to rotary motion over a substantial area of the field, the rotation being 
about a single axis. They would not, strictly, be curl $\mathbf{V}$ detectors because the area $\Delta a$ in equation (4) would be roughtly $1-2$ deg in diameter rather than being vanishingly small. Such detectors would be stimulated weakly, if at all, by Julesz and Hesse's pattern. Retinal flow patterns involving multiple, closely-spaced centres of rotation are not, however, commonly produced by self-motion through everyday environments. On the other hand, local rotaty motion about a single axis in the retinal image can be produced, for example, when an observer fixates an external object at an angle to his or her direction of motion.

\section{SECTION 5}

\section{Figure-ground extraction by motion alone}

Objects that are matched to the background in luminance, colour and texture are invisible in monocular vision when stationary, but may be clearly visible when relative motion between object and background is created either by the object's motion across the background or by the observer's motion through the environment. The object's boundaries are then defined exclusively by relative motion, either by shearing motion parallel to the boundary or by relative motion perpendicular to the boundary or by both. Figure-ground segregation may also be aided by differences in apparent depth caused by relative motion (Rogers and Graham, 1979a, b). Considerable attention has been paid to figure-ground segregation for objects whose edges are defined by luminance contrast, but comparatively little is known about figure-ground segregation for objects whose boundaries are defined by relative motion (Anstis, 1970; Regan and Spekreijse, 1970; Julesz, 1971; Braddick, 1974; Bell and Lappin, 1973; Baker and Braddick, 1982a, b; Chang and Julesz, 1983). The extensive work on the fly by Reichardt and his colleagues is the chief basis for our understanding of this problem (Reichardt and Poggio, 1979; Poggio et al., 1981; Reichardt et al., 1983; Thorson, 1966; Foster, 1971).

An experimental study on human vision compared detection thresholds for rectangular targets defined either by relative motion alone or by luminance contrast alone (Regan and Beverley, 1984b). The first kind of target consisted of a rectangular area within a 5 deg (vertical) $\times 6.3 \mathrm{deg}$ area containing about 3000 dots of $1 \mathrm{~min}$ arc diameter. The rectangular area was visible when the dots within it exceeded a threshold speed, but otherwise could not be distinguished from the surrounding dots. The second kind of target consisted of a uniformly illuminated rectangle of luminance $(L+\Delta L)$ $\mathrm{cd} / \mathrm{m}^{2}$ superimposed on a uniformly illuminated $5 \mathrm{deg}$ (vertical) $\times 6.3 \mathrm{deg}$ area of luminance $L$ $\mathrm{cd} / \mathrm{m}^{2}$. The rectangular area was visible when $\Delta L$ exceeded a threshold value. All targets were presented at 1.5 deg eccentricity.

For targets whose boundaries were defined by luminance contrast, the spatial summation area was estimated as $0.02 \mathrm{deg}^{2}$, but for motioncontrast targets the spatial summation area was considerably larger at about $0.16 \mathrm{deg}^{2}$. For targets whose boundaries were defined by luminance contrast, temporal summation followed the familiar Bloch's law plot, and was fit by a single R-C stage of temporal integration with a time constant of $61 \mathrm{msec}$. On the other hand, for targets whose boundaries were defined by relative motion, the data points could not be fit by a single integration stage. They were fitted by two cascaded stages, the first with the same $61 \mathrm{msec}$ time constant followed by a stage with the much longer time constant of $750 \mathrm{msec}$. It is interesting that the Reichardt-Poggio model of fly vision involves a running average element with a time constant of about $400 \mathrm{msec}$.

For targets defined by relative motion, retinal eccentricity had little effect on temporal integration; there was only a $40 \%$ change in time constant between 0 and $16 \mathrm{deg}$ eccentricity, though threshold rose by about 30 times. The situation was quite different for spatial summation. For a target of constant area, log threshold was closely proportional to eccentricity to at least 32 deg eccentricity; the larger the target, the less the effect of eccentricity. This log-linear proportionality is also found for contrast sensitivity when a target of constant area is used (Robson and Graham, 1981; Koenderink et al., 1978; Regan and Beverley, 1983).

In summary, spatial summation extends to considerably larger areas, and temporal summation extends to much longer durations for objects whose boundaries are defined by relative motion than for objects whose boundaries are defined by luminance contrast. The Reichardt and Poggio model can probably account for our data on figure-ground segregation by relative motion. Whether it could also account for the data on figure-ground segregation by luminance contrast has yet to be established. 
In everyday vision both relative motion and luminance contrast cues are often available as cues for figure-ground segregation. It is possible that, by identifying and localising object boundaries, the relative motion cue might usefully disambiguate luminance contrast information when the eye attempts to achieve figure-ground segregation in low-contrast scenes. In particular, rough physiological equivalents of $\operatorname{div} \mathbf{V}$ detectors (see above) might be useful in identifying object boundaries; the component of relative motion perpendicular to the boundary would produce a local maximum of $\operatorname{div} \mathrm{V}$ along the boundary. At an empirical level, however, little is known about interactions between these relative motion and luminance contrast cues to boundaries when both are present. In this context it is interesting that, even though spatial summation fields seem to be large (typically $2 \mathrm{deg}$ ), the edges of an extended target can, nevertheless, appear remarkably sharp so that good shape discrimination is possible for a $1 \mathrm{deg}$ diameter target and objects larger than about $0.5-1$ deg appear crisply defined. This paradoxical finding could be understood if edge sharpness is determined by opponency between elements driven from different retinal locations so that edge sharpness would not be limited by the summation field size of these elements, but rather by their noise levels. An interesting feature of such a hypothetical opponent-location mechanism is that, although it could provide crisp edge definition for large isolated targets it would not necessarily give a correspondingly high acuity for periodic targets such as a grating.

Acknowledgements - This work was sponsored by the U.S. Air Force Office of Scientific Research under grant AFOSR84-0030 and by the Natural Sciences and Engineering Research Council of Canada under grant A-0323. I thank $\mathrm{J}$ anet Lord for assistance in preparing this manuscript.

\section{REFERENCES}

Anstis S. M. (1970) Phi movement as a subtraction process. Vision Res. 10, 1411-1430.

Baker C. L. Jr and Braddick O. J. (1982a) Does segregation of differently moving areas depend on relative or absolute displacement? Vision Res. 22, 851-856.

Baker C. L. Jr and Braddick O. J. (1982b) The basis of area and dot number effects in random dot motion perception. Vivion Res. 22, 1253-1259.

Barlow H. B. and Levick W. R. (1965) The mechanism of directionally selective units in rabbit's retina. II: Directionally selective units. J. Physiol, Lond. 178, 477-504.
Bell H. H. and Lappin J. S. (1973) Sufficient conditions for the discrimination of motion. Percept. Psychophys. 14, 45-50.

Beverley K. I. and Regan D. (1973) Evidence for the existence of neural mechanisms selectively sensitive to the direction of movement in space. J. Physiol. 235, 17-29.

Beverley K. I. and Regan D. (1975) The relation between discrimination and sensitivity in the perception of motion in depth. J. Physiol. 249, 387-398.

Beverley K. I. and Regan D. (1979a) Visual perception of changing size: the effect of object size. Vision Res. 19. 1093-1104.

Beverley K. I. and Regan D. (1979b) Separable aftereffects of changing size and motion in depth: Different neural mechanisms? Vision Res. 19, 727-733.

Beverley K. I. and Regan D. (1980) Visual sensitivity to the shape and size of a moving object: implications for models of object perception. Perception 9, 151-160.

Braddick O. J. (1974) A short range process in apparent motion. Vision Res. 14, 519-527.

Braunstein M. L. (1976) Depth Perception Through Motion. Academic Press, New York.

Bridgeman B. (1972) Visual receptive fields sensitive to absolute and realtive motion during tracking. Science 178 , 1106-1108.

Burns B. D., Gassanov V. and Webb A. C. (1972) Responses of neurons in the cat visual cerebral cortex to relative movement patterns. J. Physiol. 226, 133-151.

Chang J. J. and Julesz B. (1983) Displacement limits, directional anisotrophy and direction versus form discrimination in random-dot cinematograms. Vision Res. 23, 639-646.

Emerson R. C. and Gerstein G. L. (1977) Simple striate neurons in the cat. II. Mechanisms underlying directional asymmetry and directional selectivity. J. Neurophysiol. $\mathbf{4 0 ,}$ 136-155.

Erkelens C. and Collewijn H. (1985) Motion perception during dichoptic viewing of moving random dot stereograms. Vision Res. 25, 583-588.

Fieandt K. and Gibson J. J. (1959) The sensitivity of the eye to two kinds of continuous transformation of a shadow pattern. J. exp. Psychol. 57, 344-347.

Foster D. H. (1971) A model of the human visual system in its response to certain classes of moving stimuli. Kybernetik 8, 69-84.

Frost B. J. and Nakayama K. (1983) Single visual neurons code opposing motion independent of direction. Science 13, 744.745 .

Gibson J. J. (1950) The Perception of the Visual World. Houghton Mifflin, Boston, Mass.

Golomb B., Anderson R. A., Nakayama K., MacLeod D. and Wong A. (1985) Visual thresholds for shearing motion in monkey and man. Percept. Psychophys.

Goodwin A. W. and Henry G. H. (1975) Direction selectivity of complex cells in a comparison with simple cells J. Neurophysiol. 38, 1524-1540.

Goodwin A. W., Henry G. H. and Bishop P. O. (1975) Direction selectivity of simple striate cells: properties and mechanism. J. neurophysiol. 38, 1500-1523.

Gordon D. A. (1965) Static and dynamic fields in human space perception. J. opt. Soc. Am. 55, 1296-1303.

Helmholtz H. von (1925) Treatise on Physiological Optics (Edited by Southall J, P. C.), Vols I-3. Dover, New York (originally published 1866).

Hoyle F. (1960) The Black Cloud, pp. 26-27. Penguin. Harmondsworth, England. 
Johansson G. (1964) Perception of motion and changing form. Scand. J. Psychol. 5, 181-208.

Julesz B. (1971) Foundations of Cyclopean Perception. Univ. of Chicago Press, Chicago.

Julesz. B. and Hesse R. I. (1970) Inability to perceive the direction of rotation of line segments. Nature 225 , 243-244.

Kaufman L. (1974) Sight and Mind, pp. 375-383. Oxford Univ. Press.

Koenderink J. J. and van Doorn A. J.(1976) Local structure of movement parallax of the plane. J. opt. Soc. Am. 66, $717-723$.

Koenderink J. J. and van Doorn A. J. (1981) Exterospecific component of the motion parallax field. J. opt. Soc. Am. 71, 953-957.

Koenderink J. J., Bouman M. A., Bueno de Mesquita A. E. and Slappendel S. (1978) Perimetry of contrast detection thresholds of moving spatial sinewave patterns. II. The far peripheral visual field. J. opt. Soc. Am. 68, 850-854.

Kruk R. and Regan D. (1983) Visual test results compared with flying performance in telemetry-tracked aircraft. Aviat. Spact Environ. Med. 54, 906-911.

Kruk R., Regan D., Beverley K. I. and Longridge T. (1983) Flying performance on the Advanced Simulator for Pilot Training and laboratory tests of vision. Human Factors $25,457-466$.

Lee D. N. (1976) A theory of visual control of braking based on information about time to collision. Perception 15, 437-459.

Levinson E. and Sekuler R. (1975) Inhibition and disinhibition of direction-specific mechanisms in human vision. Nature 254, 692-694.

Llewellyn K. R. (1971) Visual guidance of locomotion. $J$. exp. Psychol. 91, 245-261.

Longuet-Higgins H. C. and Prazdny K. (1980) The interpretation of a moving retinal image. Proc. $R$. Soc. Lond. B 208, 385-397.

MacLeod D. I. A., Nakayama K., Silverman J, and Mulligan J. B. (1983) Anisotropic spatial integration in the detection of movement. Invest. Ophthalmol. visual Sci., Suppl. ARVO Abst. 24, 277.

Marr D. and Poggio T. (1979) A computational theory of human stereo vision. Proc. R. Soc. Lond. B 204, 30l-328.

Maturana H. R. and Franck S. (1963) Directional movement and horizontal edge detectors in the pigeon retina. Science 142, 977-979.

Michael C. R. (1966) Receptive fields of directionally selective units in the optic nerve of the ground squirrel. Science 152, 1092-1095.

Miezen F., McGuinness E. and Allman J. (1982) Antagonistic direction specific mechanisms in area MT in the owl monkey. In Abstracts of the Society for Neuroscience, 12th Annual Meeting, Minneapolis.

Motter B. C. and Mountcastle V. B. (1981) The functional properties of the light sensitive neurons of posterior parietal cortex studied in waking monkeys: foveal sparing and opponent vector organization. J. Neurosci. 1, 3-26.

Movshon J. A., Thompson I. D. and Tolhurst D. J. (1978) Receptive field organization of complex cells in the cat's striate cortex. J. Physiol., Lond. 283, 79-99.

Nakayama K. and Tyler C. W. (1981) Psychophysical isolation of movement sensitivity by removal of familiar position cues. Vision Res. 21, 427-433.

Piéron H. (1931) La Sensation chromatique données sur la latence propre de l'établissement des sensations de couleur. Année Psychol. 32, 1-29.

Poggio T., Reichardt W. and Hausen K. (1981) A neuronat circuitry for relative movement discrimination by the visual system of the fly. Naturwissenschaften 68,443 466.

Priest H. F. and Cutting J. E. (1985) Visual flow and direction of locomotion (Technical Comment): Rebuttal. Science 227, 1063.

Regan D. (1982) Visual information channeling in normal and disordered vision Psychol. Rev. 89, 407-444.

Regan D. (1985) Visual flow and direction of locomotion (Technical Comment): Reply. Science 227, 1063.

Regan D. and Beverley K. I. (1973) The dissociation of sideways movements from movements in depth: psychophysics. Vision Res. 13, 2403-2415.

Regan D. and Beverley K. I. (1978a) Looming detectors in the human visual pathway. Vision Res. 18, 415-421.

Regan D. and Beverley K. I. (1978b) Illusory motion in depth: aftereffect of adaptation to changing size. Vision Res. 18, 209-212.

Regan D. and Beverley K. I. (1979a) Binocular and monocular stimuli for motion-in-depth: changingdisparity and changing-size inputs feed the same motionin-depth stage. Vision Res. 19, 1331-1342.

Regan D. and Beverley K. I. (1979b) Visually-guided locomotion: psychophysical evidence for a neural mechanism sensitive to flow patterns. Science 205, 311-313.

Regan D. and Beverley K. I. (1980) Visual responses to changing size and to sideways motion for different directions of motion in depth: linearization of visual responses. J. opt. Soc. Am. 11, 1289-1296.

Regan D. and Beverley K. I. (1982) How do we avoid confounding the direction we are looking with the direction we are moving? Science 215, 194-196.

Regan D. and Beverley K. I. (1983) Visual fields described by contrast sensitivity, by acuity and by relative sensitivity to different orientations. Invest. Ophthal. visual Sci. 24, 754-759.

Regan D. and Beverley K. I. (1984a) Psychophysics of visual flow patterns and motion in depth. In Sensory Experience, Adaptation and Perception (Edited by Spillman L. and Wooten B. R.), pp. 215-240. Erlhaum, Hillsdale, N.J.

Regan D. and Beverley K. 1. (1984b) Figure-ground segregation by motion contrast and by luminance contrast. J. opt. Soc. Am. 1, 434-442.

Regan D. and Beverley K. I. (1985) Visual responses to vorticity and the neural analysis of optic flow. $J$. opt. Soc. Am. A 2.

Regan D., Beverley K. I. and Cynader M. (1979) The visual perception of motion in depth. Scient. Am. 241, 136-151.

Regan D., Collewijn H. and Erkelens C. (1985) Necessary conditions for motion in depth perception. Invest. Ophthal. visual Sci., Suppl. 26, 242.

Regan D. and Cynader M. (1979) Neurons in area 18 of cat visual cortex selectively sensitive to changing size: nonlinear interactions between responses to two edges. Vision Res. 19, 699-711.

Regan D., Erkelens C. and Collewijn H. (1985a) Necessary conditions for the perception of motion in depth. Invest. Ophthal. visual Sci.

Regan D., Erkelens C. and Collewijn H. (1985b) Visual field defects for vergence eye movements and stereomotion perception. Invest. Ophthal. visual Sci. 
Regan D. and Spekreijse, H. (1970) Electrophysiological correlate of binocular depth perception in man. Nature 255, 92.94

Reichardt W. and Poggio T. (1979) Figure-ground discrimination by relative movement in the visual system of the fly. Biol. Cybernet. 35, 81-100.

Reichardt W., Poggio T and Hausen K. (1983) Figureground discrimination by relative movenent in the visual system of the fly. Part II: Towards the neural circuitry. Biol. Cybernet. 46, I.

Richards W. and Lieberman H. R. (1982) Velocity blindness during shearing motion. Vision Res. 22, $97-100$.

Richards W. and Regan D. (1973) A stereo field map with implications for disparity processing. Invest. Ophthal risual Sci. 12, 904-909.

Robson J. G. and Grahman N. (1981) Probability summations and regional variation in contrast sensitivity a across the visual field. Vision Res. 21, 409-418.

Rogers B. and Graham M. (1979a) Motion parallax as an independent cue for depth perception. Percept. Psychophys. 8, 125-134.

Rogers B. and Graham N. (1979b) Motion parallax as an independent cue for depth perception. l'ision Res. 22. 26I-270.

Rutherford D. E. (1954) Vector Mcthods. Interscience, New York.

Schey H. M. (1973) Dit, Grad, Curl and All That: An Informal Text on Vector Calculus. Norton. New York.

Sekuler R., Pantle A. and Levinson E. (1978) Physiological basis of motion perception. In Handhook of Sensory. Physiology (Edited by Held R.. Leibowity H. W. and Teuber H.-L.), Vol. 8. Springer. Berlin.

Stromeyer C. F. III, Kronauer R. E. and Madsen J. C. (1984) Opponent-movement mechanisms in human vision. J. opt. Soc. Am. A 1, 876-884.

Thorson J. (1966) Small signal analysis of a visual reflex in the locust: II. Frequency dependence. Kithernetik 3, 53-66.

Ullman S. (1979) The Interpretation of Visual Motion. MIT Press, Boston, Mass.

Wallach H. and O'Connell D. N. (1953) The kinetic depth effect. J. exp. Physchol. 45, 205-217.

Wheatstone C. (1852) Contributions to the physiology of vision II. Phil. Trans. R. Soc. 142, 1-18. 